\title{
ADIPOKINES BETWEEN OBESITY AND OSTEOARTHRITIS
}

\author{
Mihaela-Ana Nicolau \\ Postgraduate, Carol Davila University of Medicine and Pharmacy, Bucharest
}

\begin{abstract}
WAT (white adipose tissue) is considered nowadays a real endocrine organ, which releases an increased number of biomarkers, known as adipokines. These ones have been closely examined during the last decade, as they play an important role in the cartilage homeostasis and they can serve the early diagnosis and the treatment of osteoarthritis.
\end{abstract}

Keywords: osteoarthritis, biomarkers, adipokines, diagnosis, treatment

Osteoarthritis (OA) is a multifactorial etiology disease, characterized by the degeneration of the joint hyaline cartilage, compression of the subchondral bone and the formation of osteophyte.

The synovial inflammatory process accompanies the above mentioned processes and contributes to the symptomatology and the progression of the disease, but the inflammation has a lower intensity than the one which is present in the chronic inflammatory rheumatism.

The etiology of the OA is mostly unknown, including genetic and non-genetic factors, the most important risk factors being: age, obesity, sex, etc.

The joint cartilage is avascular, aneural, being formed of an extracellular matrix and of chondrocytes, and it represents an active metabolism, consisting of two processes: anabolic and catabolic, inside the cartilage homeostasis.

The coexistence of obesity and OA has increased considerably nowadays, being recognized that obesity contributes both at the initiation and at the development of OA, because of the mechanic stress at the joints level, under the action of the excess weight, as it is the case of the OA in the knee.

But the fact that there is a positive association between obesity and OA at the level of the hand joint implies the existence of some systemic factors, which connect the two elements of this relation. These factors are the adipokines (AK), that is some pro-inflammatory cytokines secreted by the adipocytes in the white adipose tissue (WAT), especially the one located on the abdomen, but also the infrapatellar one IPFP (infrapatellar fat pads). Adipokines may also be produced by the synovium, chondrocytes, osteoblasts and by the osteoclasts (1).

The AK have multiple roles, for example:

- they can serve as biomarkers for the early diagnosis of OA;

- they allow the assessment and the severity of $\mathrm{OA}$ and they monitor the progression of the disease;

- several pharmacological interventions can be tested.

Thus, obesity also represents a risk factor for nonweight-bearing joints, such as those in the fingers, hand and wrist, suggesting the fact that metabolic factors contribute to a higher prevalence of OA in the case of obese people (2).

Today, AK represent growth factors, being involved both in the glucose and lipid metabolism, but also in the immune and inflammatory response and they can make the connection between obesity, inflammation and OA.

AK have several important roles in the physiopathology of rheumatoid diseases and that is why an attempt is being made to clarify the connection between WAT, inflammatory disorders and metabolic diseases. 
In order to neutralize the unpleasant effects of the pro-inflammatory status in obesity, there are the following preventive strategies: reducing fat in the diet and increasing physical activity.

WAT releases cytokines such as: IL-6, IL-1 and TNF- $\alpha$, as well as AK, such as: leptin, adiponectin, resistin, visfatin, but also other factors recently identified as: lipocalin, chemerin, or serum amiloyd 3 (SAA3) $(4,5)$.

Many recent studies confirm the role of AK as a non-mechanic connection between obesity and OA.

The pathogenesis of obesity associated with OA is not currently completely understood, but recent studies state that pro-inflammatory metabolic factors contribute to a higher risk of OA.

In this context there are two questions which appear: does obesity cause local inflammation in the case of the knee, for example, by changing the load distribution and by growing the joint stress or is it the lack of this load that increases metabolic inflammation and joint susceptibility for cellular oxidative stress and for extracellular matrix degradation?

\section{TYPES OF ADIPOKINES AND THEIR INVOLVEMENT IN THE PATHOGENESIS OF OA}

The main source of all the pro-inflammatory adipokines is the dysfunction of the adipose tissue.

Leptin has been studied as a main mediator, because of the possibility to intervene in the metabolic disorders. The cloning of leptin in 1994 has opened new perspectives in the field of metabolism. It is a polypeptide hormone $16-\mathrm{kDa}$, encoded by the obesity gene, secreted mainly by adipocytes.

According to some studies, leptin increases the expression of the anabolic growth factors, such as IGF-1 (insulin like growth factor-1) and TGF- $\beta$ (transforming growth factor- $\beta$ ) (6).

The cartilage is subjected to mechanical stress, compression and hydrostatic pressure (7), all these signals being felt by the chondrocytes, which maintain the tissue homeostasis by means of the balance between the anabolic growth factors, the catabolic cytokines and the mediators of the inflammation (8).

Leptin plays an important role in the physiopathology of the OA, some studies suggesting the anabolic role of this hormone on the cartilage. Thus, a greater amount of leptin has been found in the osteoarthritic human cartilage than in normal cartilage.

Leptin concentration in the synovial fluid is $3-11$ times bigger than the plasmatic one (9) and it has been positively related to BMI (body mass index) in the case of people with severe OA(6). Consequently, it has been suggested that high circulating levels of leptin in the case of obese individuals can protect the cartilage from arthritic degeneration. However, these results are not eloquent and studies on animals will clarify in the future if leptin promotes or reduces radiographic joint lesions in arthritis.

Serum leptin levels are correlated with the decrease of radiographic joint lesions in rheumatoid arthritis (RA), thus making this AK become a possible therapeutic target in autoimmune diseases, according to Matarese et el.

It has been suggested that leptin might have a role in SLE (systemic lupus erythematosus), especially in the case of patients suffering from SLE, who have a high cardio-vascular risk.

The production of leptin in the subchondral bone osteoblasts in the case of osteoarthritis is higher than in normal cells and it may lead to the increased production of alkaline phosphatase (ALP), TGF- $\beta$ (transforming growth factor $\beta$ ), osteocalcin (OC) and type I collagen (10).

All the adipokines have greater levels in the hip joint synovial fluid than in that of the knee, besides leptin, which is found in greater quantities in the knee joint (11), being mainly produced by the infrapatellar fat pad (IPFP) (9). (Table 1)

Resistin is a pro-inflammatory mediator also known as RELM (resistin-like molecules), secreted by the adipose tissue, but also by the macrophages and the neutrophils (12).

Serum levels of resistin are not different in patients who suffer from OA compared to the control group and these are higher for patients suffering from RA than for patients suffering from OA.

The role of resistin has been studied in patients suffering from joint injury and it has been observed that resistin levels increase at the systemic and local level immediately after a joint injury (13), and they decrease after a certain period of time (14), this fact showing that resistin has a direct effect on the cartilage matrix and the production of cytokines. (Table 2)

Adiponectin is a 244-residue protein, it represents $0.01 \%$ of the total plasma protein (1), being found in great quantities in the circulating blood stream, under different molecular forms (15), being produced especially by WAT, having both pro-inflammatory and anti-inflammatory properties. 


\section{TABLE 1}

\begin{tabular}{|l|l|l|}
\hline Adipokine & Methods & Results \\
\hline $\begin{array}{l}\text { Leptin } \\
\text { (according to a study by Terlain et al) }\end{array}$ & Injecting leptin to rats. & $\begin{array}{l}\text { Leptin was strongly expressed in the bone } \\
\text { and the osteoarthritis cartilage. }\end{array}$ \\
\hline $\begin{array}{l}\text { Leptin } \\
\text { (according to a study by Pallu et al) }\end{array}$ & $\begin{array}{l}\text { Isolated chondrocytes from patients with } \\
\text { osteoarthritis, having different BMI, were } \\
\text { treated with } 20,100 \text { or } 500 \mathrm{ng} / \mathrm{ml} \text { leptin. }\end{array}$ & $\begin{array}{l}\text { At } 20 \mathrm{ng} / \mathrm{ml} \text { leptin was unable to modulate } \\
\text { the genetic expression in the chondrocytes, } \\
\text { with the exception of MMP-13 in the case of } \\
\text { obese patients with osteoarthritis. }\end{array}$ \\
\hline $\begin{array}{l}\text { Leptin } \\
\text { (according to a study by Stannus et al) }\end{array}$ & $\begin{array}{l}\text { The thickness of the knee cartilage was stu- } \\
\text { died in the case of } 163 \text { patients. }\end{array}$ & $\begin{array}{l}\text { Serum leptin levels were negatively associ- } \\
\text { ated either with the thickness of the knee } \\
\text { cartilage or with BMI. }\end{array}$ \\
\hline $\begin{array}{l}\text { Leptin } \\
\text { (according to a study by Massengale } \\
\text { et al) }\end{array}$ & $\begin{array}{l}\text { A group of } 1056 \text { people was evaluated con- } \\
\text { cerning the connection between hand oste- } \\
\text { oarthritis and the serum leptin levels. }\end{array}$ & $\begin{array}{l}\text { No association was observed between se- } \\
\text { rum leptin and hand osteoarthritis. }\end{array}$ \\
\hline $\begin{array}{l}\text { Leptin } \\
\text { (according to a study by Lubbeke et al) }\end{array}$ & $\begin{array}{l}\text { Leptin concentration and pain were evalu- } \\
\text { ated for a total number of } 219 \text { hip arthritis } \\
\text { and knee arthritis which suffered surgical } \\
\text { treatment. }\end{array}$ & $\begin{array}{l}\text { Leptin levels in the synovial fluid were signi- } \\
\text { ficantly associated with the increase of the } \\
\text { pain level, especially in the case of obese } \\
\text { women. }\end{array}$ \\
\hline
\end{tabular}

\section{TABLE 2}

\begin{tabular}{|l|l|l|}
\hline Adipokine & Methods & Results \\
\hline $\begin{array}{l}\text { Resistin } \\
\text { (according to a study by Koskinen et al) }\end{array}$ & $\begin{array}{l}\text { The study concerned the synovial fluid of } \\
88 \text { patients with knee arthritis which was } \\
\text { treated by means of surgery. }\end{array}$ & $\begin{array}{l}\text { with metalloproteinases, but not with BMI. } \\
\text { wesistin levels were correlated }\end{array}$ \\
\hline
\end{tabular}

Plasma levels of adiponectin are significantly higher in the case of women than in the case of men (16), being negatively related with BMI (body mass index), lower in obese people and higher once weight is lost (17).

Adiponectin intensifies fatty acids oxidation and it diminishes the synthesis of glucose in the liver.

Compared to other adipokines, plasma levels of adiponectin were lower in the case of patients suffering from osteoarthritis than in the case of healthy individuals and the adiponectin levels found were 100 times higher in the case of those suffering from OA than in their synovial fluid (18).

In the case of patients suffering from severe knee arthritis, compared to the control group, levels of plasma adiponectin were significantly higher, whatever the age, sex, or BMI (body mass index) (19). Frommer et al have recently proved the pro-inflammatory role of adiponectin in RA. After investigating the role of adiponectin in RA, a conclusion has been drawn that the levels of this hormone are correlated with the severity of the disease. Patients with RA and low levels of visceral fat are the ones who have the highest levels of adiponectin and radiographic joint lesions, according to Giles et al.

Adiponectin acts by means of two receptors, one of them being known as AdipoR1, which is found in the striated muscle, and the other, AdipoR2, found in the liver.
Both adiponectin and its receptors have been identified in human chondrocytes (9).

There is evidence according to which adiponectin has various effects on certain diseases with inflammatory components, such as: metabolic syndrome, cardiovascular disease, type 2 diabetes and osteoarthritis (20).

It is known that adiponectin has a protective role in cardiovascular diseases, but it may also act as a pro-inflammatory factor at the joint level, taking part in the matrix degradation.

Adiponectin has a pro-inflammatory role through the activation of NO (nitric oxide), PGE2 (prostaglandin E2), VEGF (vascular endothelial growth factor), MCP-1 (monocyte chemotactic protein I), MMP-1, MMP-3, MMP-9, MMP-13 (matrix metalloproteinase), IL-6, IL-8 $(21,22)$, and it may produce the stimulation of VCAM-1 (vascular cell adhesion molecule 1), this fact pointing the role that it has in the cartilage impairment by means of joint inflammation and leukocyte infiltration.

The role of adiponectin in OA is a controversial one. Some clinical information state the fact that adiponectin can protect against cartilage impairment and against the onset of OA.

Some researchers, in a recent study, indicate an inverse correlation between plasma adiponectin and the radiological severity of knee osteoarthritis (23). 
At the cartilage level, adiponectin intensifies chondrocyte proliferation, it stimulates the synthesis of type II and type X collagen, of proteoglycans and it increases the matrix mineralization (24).

Also, adiponectin stimulates the proliferation of osteoblasts (25), it increases the RANKL (receptor activator of nuclear factor kappa-B ligand) and it inhibits the production of OPG (osteoprotegerin) in the osteoblasts, which will further activate osteoclasts (26).

The protective role against cartilage deterioration is suggested by the fact that adiponectin may intensify the release of anti-inflammatory molecules, such as: IL-10 (interleukin-10) and IL-1 $(27,28)$.

Certain studies state that patients who had high levels of adiponectin also registered a decrease in the risk of the progression of hand OA, which led to the suggestion that adiponectin might be a hormone with a protective function against cartilage impairment (29).

The levels of adiponectin in the IPFPs in the case of knee osteoarthritis are high, according to a study (30), and there is also an increase of IL-6 (interleukin-6).

In the case of patients who suffer from severe radiological OA, 4-5 according to the Ahlback score, other researchers (31) found high plasma levels, compared to patients who suffer from a less severe OA.

In some studies there has also been discovered a connection between the Lequesne index and the plasma levels of adiponectin.

Filkova et al found in the erosive OA, compared to the nonerosive one, greater serum levels of adiponectin.

In the case of patients with OA, the levels of adiponectin in the synovial fluid were related to the impairment of the aggrecans.

In other studies it has been suggested that adiponectin was involved in the early formation of osteophyte (32).
For patients who suffer from knee osteoarthritis, the L/A (leptin/adiponectin) ratio in the synovial fluid was suggested to indicate pain - in the case of these patients.

A low L/A ratio was connected to a diminished pain in the case of knee osteoarthritis, was the measurement was done using the MPQ-SF (McGill Pain Questionnaire-Short Form) pain scale (33).

Other authors (34) have shown that adiponectin may contribute to metabolic changes in OA.

Some studies (29) suggest the fact that adiponectin may be involved in the physiopathology of hand osteoarthritis.

In conclusion, adiponectin seems to have both catabolic and anabolic effects in the onset and the progression of osteoarthritis. (Table 3)

Visfatin, also known as PBEF (pre-B-cell colony-enhancing factor) and as Nampt (nicotinamide phosphoribosyltransferase), is a protein of $52 \mathrm{kDa}$ and approximately 471 amino acids (35).

The visfatin hormone was initially discovered in the liver, muscles and bone marrow, but it has been proved to be secreted by the visceral fat, too (36). The levels in the plasma and in the synovial fluid are associated both with inflammation and with lipid metabolism $(37,38)$. Also, the visfatin levels in the synovial fluid were increased in the case of patients who had radiographic proofs of OA through various damages, compared to patients who had less severe signs of OA.

Thus, visfatin levels in the synovial fluid in grade 4 KL-scores (Kellgren-Lawrence) were much higher than those of grade $3 \mathrm{KL}$-scores (39) and these are high in RA, too.

Visfatin increases in obesity, and the leukocytes of obese patients, especially granulocytes and monocytes, produce high quantities of visfatin, compared to lean subjects (40). Macrophages may also secrete visfatin (41).

\section{TABLE 3}

\begin{tabular}{|l|l|l|}
\hline Adipokine & Methods & Results \\
\hline $\begin{array}{l}\text { Adiponectin (according to a } \\
\text { study by Francin et al) }\end{array}$ & $\begin{array}{l}\text { The evaluation concerned adiponectin in the car- } \\
\text { tilage of healthy people and of people suffering } \\
\text { from osteoarthritis. }\end{array}$ & $\begin{array}{l}\text { Adiponectin was not obvious in the healthy car- } \\
\text { tilage, but it increased in the cartilage of people } \\
\text { with osteoarthritis. }\end{array}$ \\
\hline $\begin{array}{l}\text { Adiponectin (according to a } \\
\text { study by Honsawek et al) }\end{array}$ & $\begin{array}{l}76 \text { osteoarthritis cases and 24 cases of group con- } \\
\text { trol were investigated. }\end{array}$ & $\begin{array}{l}\text { Adiponectin plasma and synovial fluid concen- } \\
\text { tration significantly decreased with the severity } \\
\text { of the disease, leading to the conclusion that this } \\
\text { hormone might have a protective role in oste- } \\
\text { oarthritis. }\end{array}$ \\
\hline $\begin{array}{l}\text { Adiponectin, leptin, resistin } \\
\text { (according to a study by Gross } \\
\text { et al) }\end{array}$ & The synovial fluid of 35 patients was examined. & $\begin{array}{l}\text { Adiponectin can contribute to the metabolic } \\
\text { changes in osteoarthritis (IL-6; TGF- } \beta \text { ). }\end{array}$ \\
\hline
\end{tabular}


Visfatin determines the growth of MMP-3, MMP-13 (metalloproteinases), ADAMTS-4 (aggrecanase 1), ADAMTS-5 (aggrecanase 2), while the production of aggrecans decreases (42), for example: high-molecular-weight proteoglycan in the osteoarthritis chondrocytes.

It can also determine the growth of cytokines IL-1 $\beta$ (interleukin- $1 \beta$ ), IL-6, TNF- $\alpha$ (tumor necrosis factor- $\alpha$ ) (43), as well as growth of NO (nitric oxide) and of PGE2 (prostaglandin E2). The decrease in the synthesis of proteoglycans by visfatin indicates its prodegradative role on the joint cartilage.

Visfatin also produces the inhibition of the collagen type II synthesis and helps the development of osteoblasts.

Some authors (42) have shown that visfatin has a catabolic role on the cartilage and it may have and important function in the OA physiopathology.

Other researchers (39) have discovered that visfatin in the synovial fluid of patients suffering from OA may be involved in the degradation process of the matrix (39), as visfatin is positively related to the degradation biomarkers of collagen type II and aggrecan.

The role of visfatin in glucose metabolism is still unclear, but it is supposed that it might have insulin mimetic properties $(36,44)$.

Regulation of the visfatin synthesis is done by the following factors: glucocorticoids, TNF- $\alpha$ (tumor necrosis factor- $\alpha$ ), IL-6 (interleukin-6), and growth hormone (43).

IPFP (infrapatellar fat) of patients suffering from OA secretes high quantities of visfatin, according to some studies (45), and that is why this biomarker may contribute to pathophysiological changes which appear in OA. (Table 4)

\section{OTHER ADIPOKINES: CHEMERIN, LIPOCALIN 2, SAA3, VASPIN, OMENTIN AND NESFATIN}

Chemerin, also known as tazarotene-induced gene (TIG2) or as retinoic acid receptor responder 2
(RARRES2), is a recently discovered adipokine (46). It is secreted as an $18 \mathrm{kDa}$ inactive precursor and it is activated as posttranslational C-terminal cleavage (47).

Chemerin is mainly secreted by the adipose tissue (48), but also by the immune-competent cells (49). This hormone acts by means of a receptor (CMKLR1) (46) and it is an adipokine involved in the metabolic and immune homeostasis $(46,48,49)$.

Dexamethasone induces chemerin secretion, relating it to BMI (body mass index).

Chemerin may be invoved both in the development and in the progression of OA, a fact that was suggested by some researchers (50), who studied the relation between chemerin serum levels and its levels in the synovial fluid in the case of patients suffering from knee osteoarthritis.

Lipocalin 2 (LCN2), also known as siderocalin, $24 \mathrm{p} 3$, uterocalin, or neutrophil gelatinase-associated lipocalin (NGAL), is a $25 \mathrm{kDa}$ glycoprotein separated from neutrophil granules, though the adipose tissue is the main source of LCN2 (51). Lipocalin 2 is involved in: apoptosis in hematopoietic cells (52); transport of fatty acids and iron (53); metabolic homeostasis (54); modulation of inflammation (55); suppression of bacterial growth (56); regulation of iron metabolism (57).

Expression of LCN2 is altered in certain pathological conditions, as for example: obesity, adipose tissue hypoxia and anemia (57).LCN2 has recently been identified in chondrocytes, its main regulators being TNF and dexamethasone (58). This hormone might be part of the matrix degradation, as it creates molecular complexes with MMP-9 (which it protects) $(59,60)$.

The ease of LCN2 to protect MMP-9 (metalloproteinase-9) may illustrate an important mechanism by means of which LCN2 contributes to cartilage impairment in the case of OA.

Further studies will reveal the therapeutic potential of these adipokines.

LCN2 has recently been proposed as a biomarker of cartilage impairment in the case of rheumatoid arthritis.

\section{TABLE 4}

\begin{tabular}{|l|l|l|}
\hline Adipokine & Methods & Results \\
\hline $\begin{array}{l}\text { Visfatin } \\
\text { (according to a study by Gosset et al) }\end{array}$ & $\begin{array}{l}\text { The production of visfatin in human } \\
\text { chondrocytes was analysed. }\end{array}$ & $\begin{array}{l}\text { Visfatin has a catabolic role in the cartilage and } \\
\text { can have a role in the pathology of osteoarthri- } \\
\text { tis. }\end{array}$ \\
\hline $\begin{array}{l}\text { Visfatin } \\
\text { (according to a study by Chen et al) }\end{array}$ & $\begin{array}{l}\text { It examined visfatin distribution both in } \\
\text { the synovial fluid and in the serum, for a } \\
\text { group of patients with osteoarthritis and } \\
\text { in the serum of a control group. }\end{array}$ & $\begin{array}{l}\text { Visfatin serum levels were higher in patients } \\
\text { with osteoarthritis compared to the control } \\
\text { group, and the visfatin concentrations in the } \\
\text { synovial fluid surpassed the serum ones. }\end{array}$ \\
\hline
\end{tabular}




\section{SAA3 (SERUM AMYLOID A3)}

It is an adipokine which is part of the acute-phase proteins in the amyloid A serum (A-SAA). Factors like: IL-1 $\beta$, TNF, dexamethasone, IL-6, as well as certain circumstances like obesity determine the expression of SAA3 (61).The expression SAA3 has recently been described in chondrocytes, where SAA3 stayed regulated by cytokines such as: IL- $\beta$, leptin or adiponectin (62).

Vaspin (visceral adipose tissue-derived serine protease inhibitor) - is a visceral adipose tissue derived from serpin, having potential antiprotease properties (63).

Levels of vaspin in the synovial fluid were higher in the case of patients suffering from rheumatoid arthritis, compared to patients suffering from OA.

Omentin is a $40 \mathrm{kDa}$ protein, a new adipokine, secreted by the omental adipose tissue, which is found in high quantities in human plasma. This hormone can regulate insulin action (64). Plasma omentin levels decrease with obesity and they might positively be associated with plasma adiponectin and negatively associated with BMI (body mass index), waist circumference and insulin level, these being markers of the metabolic syndrome.

Recent studies state that omentin alternates between inflammation and obesity, being recently identified as intelectin, a new type of calcium-dependent lectin.

Some authors have recently discovered the decrease of omentin levels in patients with rheumatoid arthritis compared to patients with OA (65).

\section{REFERENCES}

1. Gegout P.P., Francine P.J., Mainard D. et al. Adipokines in osteoarthritis: friends or foes of cartilage homeostasis ? Joint Bone Spine 2008; 75: 669-671.

2. Carman W.J., Sowers M., Hawthorne V.M. et al. Obesity as a risk factor for osteoarthritis of hand and wrist: a prospective study, American Journal of Epidemiology, vol.139, no.2,1994; 119-129.

3. Gualillo O., Gonzalez-Juanatey J.R., Lago F. The emerging role of adipokines as mediators of cardiovascular function: physiologic and clinical perspectives, Trends in Cardiovascular Medicine, vol. 372, no. 6505, 1994; 425-432.

4. Hotamisligil G.S., Shargill N.S., Spiegelman B.M. Adipose expression of tumor necrosis factor-alfa: direct role in obesity-linked insulin resistance, Science, vol. 259, no. 5091, 1993; 87-91.

5. Fantuzzi G. Adiponectin and inflammation: consensus and controversy, Journal of Allergy and Clinical Immunology, vol. 121, no. 2, 2008; 326-330.

6. Dumond H., Presle N., Terlain B. et al. Evidence for a key role of leptin in osteoarthritis. Arthritis Rheum 2003; 48(11): 3118-29.

7. Darling E.M., Athanasiou K.A. Biomechanical strategies for articular cartilage regeneration. Ann Biomed Eng 2003; 31(9): 1114-24;
All this suggests the fact that vaspin and omentin are involved in the physiopathology of osteoarthritis.

Nesfatin is present in the articular tissue and it can contribute to the physiopathology of osteoarthritis, according to some researches (66).

\section{CONCLUSIONS}

Early diagnosis and prevention by means of biomarkers represent important stages in the management of osteoarthritis.

After several researches, it has been observed that some adipokines have a catabolic role in the case of this disease, while others have both a catabolic and an anabolic role.

Thus, all recent studies provide a new representation regarding the connection between obesity and osteoarthritis, but also a new preventive approach, which may decrease the prevalence of osteoarthritis among the population.

This publication benefited from the financial support of the project ,Programme of excellency in the multidisciplinary doctoral and post-doctoral research of chronic diseases ", contract no. POSDRU/ 159/1.5/S/133377, beneficiary ,,Gr. T. Popa“ University of Medicine and Pharmacy of Iasi, project co-financed from the European Social Fund through the Sectoral Operational Programme Human Resources Development (SOP HRD) 2007-2013.

8. Urban J.P. The condrocyte: a cell under pressure. Rheumatology 1994; 33(10): 901-8.

9. Presle N., Pottie P., Dumond H. et al. Differential distribution of adipokines between serum and synovial fluid in patients with osteoarthritis. Contribution of joint tissue to their articular production. Osteoarthritis Cartilage 2006; 14: 690-695.

10. Mutabaruka M.S., Aoulad Aissa M., Delalandre A. et al. Local leptin production in osteoarthritis subchondral osteoblasts may be responsible for their abnormal phenotypic expression, Arthritis Research and Therapy, vol. 12, no. 1, article R20, 2010.

11. Van Spil W.E., Welsing P.M., Kloppenburg M. et al. Crosssectional and predictive associations between plasma adipokines and radiographic signs of early-stage knee osteoarthritis: data from CHECK. Osteoarthritis Cartilage 2012; 20: 1278-128.

12. Steppan C.M., Lazar M.A. Rezistin and obesity-associated insulin resistance, Trends in Endocrinology and Metabolism, vol. 13, no. 1, pp. 18-23, 2002;

13. Salihu H.M., Bonnema S.M., Alio A.P. Obesity: What is an elderly population growing into? Maturitas 2009; 63: 7-12.

14. Lee J.H. et al. Resistin is elevated following traumatic joint injury and causes matrix degradation and release of inflammatory 
cytokines from articular cartilage in vitro. Osteoarthritis Cartilage 17, 2009; 613-620

15. Oh D.K., Ciaraldi T., Henry R.R. Adiponectin in health and disease, Diabetes, Obesity and Metabolism, vol. 9, no. 3, 2007; 282-289.

16. Filkova M., Liskova M., Hulejova H. et al. Increased serum adiponectin levels in female patients with erosive compared with non-erosive osteoarthritis. Ann Rheum Dis 2009; 68: 295-296.

17. Cnop M., Havel P.J., Utzschneider K.M. et al. Relationship of adiponectin to body fat distribution, insulin sensitivity and plasma lipoproteins: evidence for independent roles of age and sex. Diabetologia 2003; 46: 459-469.

18. Chen T.H., Chen L., Hsieh M.S. et al. Evidence for a protective role for adiponectin in osteoarthritis. Biochim Biophys Acta 2006 1762: 711-718.

19. De Boer T.N., van Spil W.E., Huisman A.M. et al. Serum adipokines in osteoarthritis; comparison with controls and relationship with local parameters of synovial inflammation and cartilage damage. Osteoarthritis Cartilage 2012; 20: 846-853.

20. Matsuzawa Y. Therapy insight: adipocytokines in metabolic syndrome and related cardiovascular disease, Nature Clinical Practice Cardiovascular Medicine, vol. 3, no. 1, 2006; 35-42.

21. Lago R., Gomez R., Otero M. et al. A new plyer in cartilage homeostasis: adiponectin induces nitric oxide synthase type II and pro-inflammatory cytokines in chondrocytes. Osteoarthritis Cartilage 2008; 16: 1101-1109.

22. Kang E.H., Lee Y.J., Kim T.K. et al. Adiponectin is potential catabolic mediator in osteoarthritis cartilage. Arthritis Res Ther 2010; 12: 231.

23. Honsawek S., Chayanupatkul M. Correlation of plasma and synovial fluid adiponectin with knee osteoarthritis severity, Archives of Medical Research, vol. 41, no. 8, 2010; 593-598.

24. Challa T.D., Rais Y., Ornan E.M. Effect of adiponectin on ATDC5 proliferation, differentiation and signaling pathways. Mol Cell Endocrinol 2010; 323: 282-291.

25. Luo X.H., Guo L.J., Yuan L.Q. et al. Adiponectin stimulates human osteoblasts proliferation and differentiation via the MAPK signaling pathway. Exp Cell Res 2005; 309: 99-109.

26. Luo X.H., Guo L.J., Xie H. et al. Adiponectin stimulates RANKL, and inhibits OPG expression in human osteoblasts through the MAPK signaling pathway. J Bone Miner Res 2006; 21: 1648-1656.

27. Ehling A., Schaffler A., Herfarth H. et al.The potential of adiponectin in driving arthritis. J Immunol 2006; 176: 4468-4478.

28. Tilg H., Moschen A.R. Adipocytokines: mediators linking adipose tissue, inflammation and immunity. Nat Rev Immunol 2006; 6: $772-783$.

29. Yusuf E., loan-Facsinay A., Bijsterbosch J. et al. Association between leptin, adiponectin and resistin and long-term progression of hand osteoarthritis, Annals of the Rheumatic Diseases, vol. 70, no. 7,$2011 ; 1282-1284$

30. Distel E., Cadoudal T., Durant S. et al. The infrapatellar fat pad in knee osteoarthritis: an important source of interleukin- 6 and its soluble receptor. Arthritis Rheum 2009; 60: 3374-3377.

31. Koskinen A., Juslin S., Nieminen R. et al. Adiponectin associates with markers of cartilage degradation in osteoarthritis and induces production of proinflammatory and catabolic factors through mitogen-activated proteinkinase pathways. Arthritis Res Ther 2011; 13: 184.

32. Connor J.R., Dodds R.A., Emery J.G. et al. Human cartilage glycoprotein 39 (HC gp-39) mRNA expression in adult and fetal chondrocytes, osteoblasts and osteocytes by in-situ hybridization. Osteoarthritis Cartilage 2000; 8: 87-95.

33. Gandhi R., Takahasi M., Smith H. et al. The synovial fluid adiponectin-leptin ratio predicts pain with knee osteoarthritis.Clin Rheumatol 2010; 29: 1223-1228.

34. Gross J.B., Guillaume C., Gegout-Pottie P. et al. Synovial fluid levels of adipokines in osteoarthritis: Association with local factors of inflammation and cartilage maintenance. Biomed Mater Eng 2014; 24: 17-25

35. Samal B., Sun Y., Stearns G. et al. Cloning and characterization of the cDNA encoding a novel human pre-B-cell colony-enhancing factor, Molecular and Cellular Biology, vol. 14, no.2, 1994; 1431-1437.

36. Fukuhara A., Matsuda M., Nishizawa M. et al. Visfatin: a protein secreted by visceral fat that mimics the effects of insulin, Science,vol. 307, no. 5708, 2005; 426-430.
37. Auguet T., Terra X., Porras J.A. et al. Plasma visfatin levels and gene expression in morbidly obese women with associated fatty liver disease. Clin Biochem 2013; 46: 202-208.

38. Lago F., Dieguez C., Gomez-Reino J. et al. Adipokines as emerging mediators of immune response and inflammation. Nat Clin Pract Rheumatol 2007; 3: 716-724.

39. Duan Y., Hao D., Li M. et al. Increased synovial fluid visfatin is positively linked to cartilage degradation biomarkers in osteoarthritis. Rheumatol Int 2012; 32: 985-99.

40. Catalan V., Gomez-Ambrosi J., Rodriguez A. et al. Association of increased Visfatin/PBEF/NAMPT circulating concentration and gene expression levels in peripheral blood cells with lipid metabolism and fatty liver in human morbid obesity, Nutrition, Metabolism and Cardiovascular Diseases, vol. 21, no.4, 2011; 245-253.

41. Curat C.A., Wegner V., Sengenes C. et al. Macrophages in human visceral adipose tissue: increased accumulation in obesity and a source of resistin and visfatin, Diabetologia, vol. 49, no. 4, 2006; 744-747.

42. Gosset M., Berenbaum F., Salvat C. et al. Crucial role of visfatin pre-B cell colony-enhancing factor in matrix degradation and prostaglandin E2 synthesis in chondrocytes: possible influence on osteoarthritis, Arthritis and Rheumatism,vol. 58, no. 5,2008; 1399 1409.

43. Moschen A.R., Kaser A., Enrich B. et al. Visfatin, an adipocytokine with proinflammatory and immunomodulating properties, Journal of Immunology, vol. 178, no. 3, 2007; 1748-1758.

44. Fukuhara A., Matsuda M., Nishizawa M. et al. Retraction, Science, vol.318, no. 5850, 2007; 565

45. Klein-Wieringa I.R., Kloppenburg M., Bastiaansen-Jenniskens et al. The infrapatellar fat pad of patients with osteoarthritis has an inflammatory phenotype, Annals of the Rheumatic Diseases, vol. 70, no. 5, 2011; 851-857

46. Wittamer V., Franssen J.D., Vulcano M. et al. Specific recruitment of antigen-presenting cells by chemerin, a novel processed ligand from human inflammatory fluids, Journal of Experimental Medicine, vol. 198, no. 7, 2003; 977-985

47. Zabel B.A., Allen S.J., Kulig P. et al. Chemerin activation by serine proteases of the coagulation, fibrinolytic, and inflammatory cascades, Journal of Biological Chemistry, vol. 280, no. 41, 2005; 34661-34666.

48. Bazaoglu K., Bolton K., McMillan J. et al. Chemerin is a novel adipokine associated with obesity and metabolic syndrome, Endocrinology, vol. 148, no. 10, 2007; 4687-4694.

49. Luangsay S. et al. Mouse ChemR23 is expressed in dendritic cells subsets and macrophages and mediates an anti-inflammatory activity of chemerin in a lung disease model. J. Immunol. 183, 2009; 6489-6499.

50. Huang K., Du G., Li L., Liang H. et al. Association of chemerin levels in synovial fluid with the severity of knee osteoarthritis. Biomarkers 2012; 17: 16-20.

51. Triebel S., Blaser J., Reinke H. et al. A 25 kDa alfa2microglobulin-related protein is a component of the $125 \mathrm{kDa}$ form of human gelatinase, FEBS Letters, vol. 314, no. 3, 1992; 386-388.

52. Devireddy L.R., Teodoro J.G., Richard F.A. et al. Induction of apoptosis by a secreted lipocalin that is transcriptionally regulated by IL-3 deprivation. Science 293, 2001; 829-834.

53. Yang J. et al. An iron delivery pathway mediated by lipocalin. Mol. Cell 10, 2002; 1045-1056.

54. Yan Q.W. et al. The adipokine lipocalin 2 is regulated by obesity and promotes insulin rezistance. Diabetes 56, 2007; 2533-2540.

55. Cowland J.B., Borregaard N. Molecular characterization and pattern of tissue expression of the gene for neutrophil gelatinaseassociated lipocalin from humans. Genomics 45, 1997; 17-23.

56. Goetz D.H. et al. The neutrophil lipocalin NGAL is a bacteriostatic agent that interferes with siderophore-mediated iron acquisition. Mol. Cell 10, 2002; 1033-1043.

57. Jiang W., Constante M., Santos M.M. Anemia Upregulates lipocalin 2 in the liver and serum. Blood Cells Mol. Dis. 41, 2008; 169-174.

58. Owen H.C., Roberts S.J., Ahmed S.F. et al. Dexamethasoneinduced expression of the glucocorticoid response gene lipocalin 2 in chondrocytes. Am J. Physiol Endocrinol Metab 294, 2008; 1023-1034. 
59. Gupta K., Shukla M., Cowland J.B. et al. Neutrophil gelatinaseassociated lipocalin is expressed in osteoarthritis and forms a complex with matrix metalloproteinase 9. Arthritis Rheum. 56.

60. Lin Y. et al. Hyperglycemia-induced production of acute phase reactants in adipose tissue by the intestinal microbiota. PLOS ONE 4, e5842, 2009.

61. Fasshauer M. et al. Serum amyloid $A 3$ expression is stimulated by dexamethasone and interleukin-6 in 3T3-L1 adipocytes. J Endocrinol. 183, 2004; 561-567.

62. Conde J., Gomez R. Bianco G. et al. Expanding the adipokine network in cartilage: identification and regulation of novel factors in human and murine chondrocytes, Annals of the Rheumatic Diseases, vol. 70, no. 8, 2007; 275-283.

63. Hida K., Wada J., Eguchi J. et al. Visceral adipose tissue-derived serine protease inhibitor: a unique insulin-sensitizing adipocytokine in obesity, Proceeding of the National Academy of Science of the United States of America, vol. 102, no. 30, 2005; 10610-10615.

64. Yang R.Z. et al. Identification of omentin as a novel depot-specific adipokine in human adipose tissue: possible role in modulating insulin action Am J Physiol Endocrinol Metab. 290, 2006; 1253-1261.

65. Senolt L. et al. Vaspin and omentin: new adipokines differentially regulated at the site of inflammation in rheumatoid arthritis. Ann Rheum Dis. 69, 2010; 1410-1411.

66. Jiang L., Bao J., Zhou X. et al. Increased serum levels and chondrocyte expression of nesfatin-1 in patients with osteoarthritis and its relation with BMI, hsCRP, and IL-18. Mediators Inflamm, Epub 2013. 